\title{
Clinical significance of insulin receptor substrate-I down-regulation in non-small cell lung cancer
}

\author{
CHANG HOON HAN ${ }^{1}$, JAE YONG CHO ${ }^{2}$, JONG TAE MOON ${ }^{2}$, HYUNG JUNG KIM ${ }^{2,3}$, SE KYU KIM ${ }^{2-5}$, \\ DONG HWAN SHIN ${ }^{6}$, JOON CHANG ${ }^{2,3}$, CHUL MIN AHN ${ }^{2,3}$, SUNG KYU KIM ${ }^{2,3}$ and YOON SOO CHANG ${ }^{2}$ \\ ${ }^{1}$ Department of Internal Medicine, National Health Insurance Corporation Ilsan Hospital, Koyang; ${ }^{2}$ Department of \\ Internal Medicine, ${ }^{3}$ The Institute of Chest Diseases, ${ }^{4}$ Brain Korea 21 Project for Medical Sciences, ${ }^{5}$ Cancer \\ Metastasis Research Center and ${ }^{6}$ Department of Pathology, Yonsei University College of Medicine, Seoul, Korea
}

Received April 3, 2006; Accepted June 28, 2006

\begin{abstract}
Insulin receptor substrate-1 (IRS-1) is an adaptor protein for insulin-like growth factor (IGF) signaling and it is presumed associated with cancer development, progression or clinical outcome of patients harboring solid tumors. Therefore, we investigated by immunohistochemistry, the expression of IRS-1 in the tumor tissues from 94 patients who were diagnosed as stage I non-small cell lung cancer (NSCLC) and had undergone a curative lung resection. The relationships between its intratumoral expression and various clinical parameters were explored. IRS-1 is consistently expressed in the cytoplasm of intrapulmonary bronchial and bronchiolar epithelial cells comprising normal appearing adjacent lung tissues. Forty-one (43.6\%) of 94 specimens showed loss of IRS-1 expression. In a subset analysis, IRS-1 was more frequently lost in stage IB than in IA tumors (50.0 vs. $22.7 \%$, $\mathrm{p}=0.024, \chi^{2}$ test), which was reflected by the facts that tumors which showed down-regulation of IRS-1 had larger area than those with IRS-1 expression ( 18.1 vs. $12.1 \mathrm{~cm}^{2}, \mathrm{p}=0.044$, t-test). Down-regulation of IRS-1 is more frequently observed in squamous cell carcinoma than other cell type lung cancer $\left(\mathrm{p}=0.002, \chi^{2}\right.$ test $)$ and its expression was not affected by histological grade of differentiation. Comparing pack-years (P.Y.) between groups of smokers whose tumor expressed IRS-1 and those that did not, smokers whose tumor showed loss of IRS-1 expression had higher P.Y. than those whose tumor did express IRS-1 (39.2 \pm 23.67 vs. $25.6 \pm 26.61$ P.Y., $\mathrm{p}=0.034$, t-test). Intratumoral expression of IRS- 1 did not influence disease-free survival, disease-specific survival or overall survival of stage I NSCLC patients, whose median follow-up duration is 7.5 years (95\% CI; 7.21-7.86 years).
\end{abstract}

Correspondence to: Dr Y.S. Chang, Department of Internal Medicine, Yonsei University College of Medicine, 8th floor, Annex building, 146-92 Dogok-Dong, Kangnam-Ku, Seoul 135-720, Korea E-mail: yschang@yumc.yonsei.ac.kr

Key words: insulin-like growth factor, insulin receptor substrate-1, non-small cell lung cancer
These results suggest that loss of IRS-1 might rather be an early event in NSCLC development than a prognostic factor and that it is more strongly related with squamous cell carcinoma and with smoking.

\section{Introduction}

Despite years of intense efforts to control lung cancer mortality with surgical resection, chemotherapy and radiotherapy, it remains the most common cause of cancer death worldwide. This dismal outlook clearly indicates urgent need for elucidation of the mechanisms of lung cancer carcinogenesis, as well as new approaches for prevention and treatment.

Insulin-like growth factors (IGFs) are synthesized by almost all tissues and regulate cell proliferation, differentiation and apoptosis, all important attributes to tumorigenesis (1). Several studies on cancer risks have suggested that high levels of circulating IGF-1 are associated with increased risk of solid cancers including non-small cell lung cancer (NSCLC) (2-5). Binding of IGF-1 to its receptor (IGF-1R) results in receptor autophosphorylation and subsequent phosphorylation of Shc and insulin receptor substrate (IRS) $-1,-2,-3$ and -4. (6).

IRS-1, which is critical to IGF signaling, belongs to the IRS family of adaptor molecules and is tyrosine-phosphorylated in response to IGF-1, insulin and other cytokines $(7,8)$. Phosphorylation of $\mathrm{Tyr}^{608}$ and $\mathrm{Tyr}^{628}$ of IRS-1 (using rat IRS-1 numbering) generates the major docking sites for phosphatidyl inositol 3-kinase (PI3K) and activates multiple signaling pathways, including PI3K, mitogen-activated protein kinase (MAPK) and Akt (9). Activation of these signaling pathways induces multifunctional biologic actions of IGFs, including cell growth, differentiation and survival. On the other hand, serine/threonine phosphorylation of 50 potential sites of IRS-1 is regarded to oppose the biologic activity of tyrosine phosphorylation (8). Besides phosphorylation of IRS-1, chronic exposure to IGFs, mannitol, okadaic acids, high glucose and hyperinsulinemia decreased the cellular level of IRS-1, which blunted response to apoptotic signals (10). Aberrant expression of IRS-1 has been associated with the pathogenesis of many diseases, including diabetes and cancer of the breast, pancreas, prostate, and liver (11-14). By extension, a delicate balance between 'positive' IRS-1 tyrosine 
Table I. Analysis of the patients with stage 1 NSCLC according to the expression status of IRS-1.

\begin{tabular}{|c|c|c|c|}
\hline & \multicolumn{2}{|c|}{ IRS-1 expression in tumor tissue } & \multirow[b]{2}{*}{ P-value } \\
\hline & Down-regulated $(n=41)$ & Expressed $(n=53)$ & \\
\hline Age $^{\mathrm{b}}$ (years) & $63.3 \pm 8.93$ & $60.6 \pm 10.59$ & 0.205 \\
\hline \multicolumn{4}{|l|}{ Gender } \\
\hline Male & 33 & 39 & 0.433 \\
\hline Female & 8 & 14 & \\
\hline \multicolumn{4}{|l|}{ Smoking status } \\
\hline Smoker; pack-year ${ }^{\mathrm{b}}$ & $24 ; 39.2 \pm 23.67$ & $26 ; 25.6 \pm 26.61$ & $0.077 ; 0.034^{\mathrm{a}}$ \\
\hline Non-smoker & 4 & 13 & \\
\hline Unknown & 13 & 14 & \\
\hline \multicolumn{4}{|l|}{ Pathology } \\
\hline Adenocarcinoma & 12 & 35 & $0.002^{\mathrm{a}}$ \\
\hline Squamous cell carcinoma & 26 & 17 & \\
\hline Other NSCLC cell type & 3 & 1 & \\
\hline \multicolumn{4}{|l|}{ Histological grade } \\
\hline Well differentiated & 13 & 17 & 0.749 \\
\hline Moderately differentiated & 10 & 19 & \\
\hline Poorly differentiated & 9 & 12 & \\
\hline Unclassified & 9 & 5 & \\
\hline \multicolumn{4}{|l|}{ TNM stage } \\
\hline T1N0M0 & 5 & 17 & $0.024^{\mathrm{a}}$ \\
\hline T2N0M0 & 36 & 36 & \\
\hline Tumor areab $\left(\mathrm{cm}^{2}\right)$ & $18.1 \pm 14.55$ & $12.1 \pm 13.76$ & $0.044^{\mathrm{a}}$ \\
\hline \multicolumn{4}{|l|}{$\begin{array}{l}\text { Mean survival time, (months), } \\
(95 \% \mathrm{CI})\end{array}$} \\
\hline Disease-free & $98.6(79.5-117.7)$ & $93.6(80.8-106.4)$ & 0.422 \\
\hline Disease-specific & $111.4(93.8-129.0)$ & $102.5(91.1-113.8)$ & 0.562 \\
\hline Overall & $109.0(91.3-126.7)$ & $97.6(86.0-109.1)$ & 0.829 \\
\hline
\end{tabular}

${ }^{\mathrm{a} p}$-value $<0.05 .{ }^{\mathrm{b}}$ Mean \pm standard devation.

phosphorylation and 'negative' serine/threonine phosphorylation with decreased IRS-1 could regulate the functions of IRS-1, and shifts of this equilibrium could lead to pathological situations (8).

Current understandings on the IGF system suggest that post-receptor signaling pathways are important in elucidating the involvement of the IGF system in lung cancer development. Previous research suggest that IRS-1 expression in NSCLC tissue might be associated with development and/ or clinical outcome of lung cancer $(15,16)$. Consequently, we evaluated IRS-1 expression in 94 stage I NSCLC tissue samples and investigated it with clinical and pathological findings.

\section{Materials and methods}

Study population. Ninety-four specimens were obtained from patients diagnosed as stage I NSCLC between 1993 and
2004. All patients had undergone a curative surgical removal of a primary lesion at the time of diagnosis and had not received either radiotherapy or chemotherapy before or after surgery until disease recurred. Histopathological diagnosis and classification of tumors was based on criteria of the World Health Organization (WHO). The post-surgical, pathological tumor-node-metastasis (TNM) stage of each malignancy was determined according to the guidelines of the American Joint Committee on Cancer. Details of clinical and pathological characteristics are presented in Table I.

Immunohistochemical staining (IHC) of IRS-1. IHC was performed using the $\mathrm{LABS}^{\circledR} 2$ system (Dako Corp., Carpinteria, CA, USA) according to the manufacturer's instructions. Briefly, paraffinized sections were dewaxed in xylene baths and rehydrated using a graded alcohol series. Following deparaffinization, sections were immersed in methanol for 


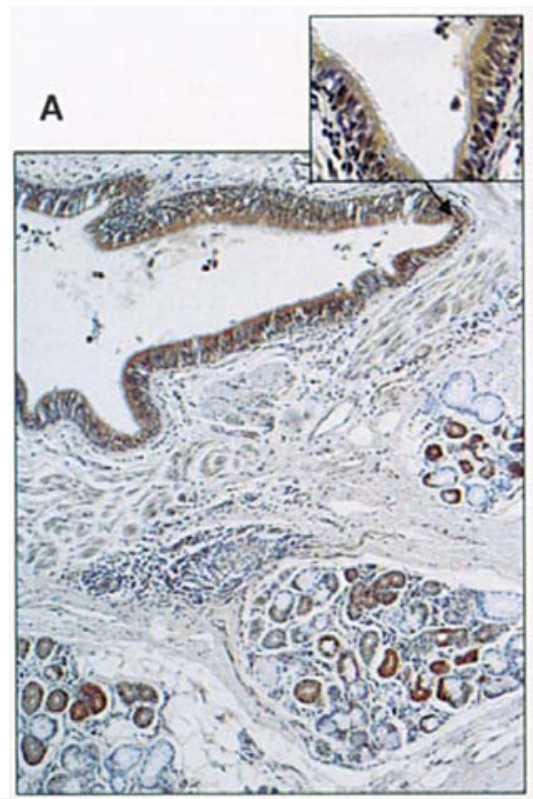

B

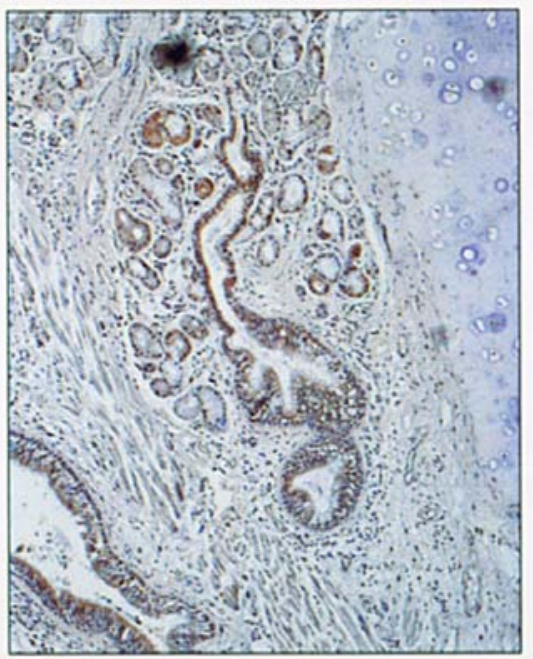

C

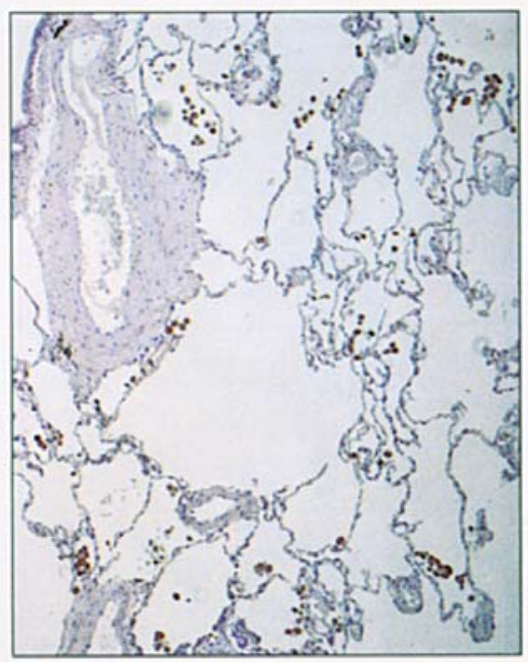

Figure 1. IRS-1 expression in normal-appearing adjacent lung tissue. Cytoplasm of epithelial cells comprising intrapulmonary bronchi (A) and bronchioles (B) showed strong and constant IRS-1 expression. Nuclei of those cells were also frequently stained with anti-IRS-1 antibody (A, inset). Other cells comprising lung tissue, including type 1 and 2 alveolar cells, vascular endothelial cells were not stained by anti-IRS-1 antibody (C). (Magnification x200; inset, $\mathrm{x} 400$ ).

5 min to block endogenous peroxidase activity and then incubated for $10 \mathrm{~min}$ with primary anti-IRS-1 antibodies (Santa Cruz Biotechnology, Santa Cruz, CA, USA) in antibody diluent (Dako Corp.) at 1:100 dilution. Sections were incubated for another $10 \mathrm{~min}$ with biotinylated linker and processed using avidin-biotin IHC techniques. Each experiment included positive and negative controls. 3,3'diaminobenzidine (DAB) was used as a chromogen in conjunction with the Liquid DAB substrate kit (Novacastra, UK). All slides were examined by light microscopy and classified by pathologists who were blinded to the relevant clinical information. Cancer cells in at least four fields were counted at x200 magnification. IRS-1 immuno-reactivity was classified by the proportion of positive cells; $0,<5 \%$ positive cells; $1+, 5-30 \%$ positive cells; $2+, 30-50 \%$ positive cells; $3+$, $>50 \%$ positive cells. Intensity of IRS-1 expression was scored as negative to weak ( 0$)$, moderate (1) or strong (2). The score is the sum of intensity and percentage of positive cells and a score $\leq 1$ was applied as a cut-off for loss of IRS-1 expression.

Statistical analysis. Survival data of all patients were obtained from the Korea National Statistical Office on June 17, 2005. In univariate analyses, independent sample $\mathrm{t}-$ and $\chi^{2}$ tests were used for continuous and categorical variables, respectively. The Kaplan-Meier estimator was used to compute the survival probability as a function of time. The log-rank test was used to compare survival times between patient groups. Overall, disease-specific and disease-free survival times were also analyzed. All statistical tests were two-tailed, and a p-value $<0.05$ was considered statistically significant.

\section{Results}

Expression of IRS-1 in adjacent normal-appearing lung tissue. To get a positive control for IHC, authors explored IRS-1 expression in NSCLC cell lines by Western blot. NCI-H157, -H358, -H596, -H1299, -H1792 and -A549 cell lines exhibited strong IRS-1 expression and, therefore, a cell block of -H1299 was included as an external positive control in each experiment with Ki-67 staining (data not shown). IRS-1 expression was prominent in the cytoplasm of normal-appearing bronchial and bronchiolar epithelial cells and localized in the perinuclear area. The intensity of IRS-1 staining in the cytoplasm was strong, granular and distinct (Fig. 1A and B). IRS-1 expression in the cytoplasm of bronchial and bronchiolar epithelial cells was constant and used as a positive internal control in this study. Nuclei of bronchial and bronchiolar epithelial cells also reacted with anti-IRS-1 antibody frequently, however, its immunoreactivity was neither homogeneous nor uniform among bronchi and bronchioles (Fig 1A, inset). Type I and II pneumocytes, endothelial cells and other cells comprising lung tissue did not react with anti-IRS-1 antibody except the cytoplasm of submucosal glandular cells (Fig. 1C).

IRS- 1 expression in NSCLC tissue and clinical and pathological parameters associated with its expression. Forty-seven (50.0\%) out of the 94 NSCLCs were adenocarcinoma, 43 (45.7\%) squamous cell carcinomas, and 4 (4.3\%) other histologic type NSCLCs. IHC analysis of NSCLC tissue samples revealed that IRS-1 expression was lost in 41 (43.6\%) NSCLC samples, using a $5 \%$ cut-off (Fig. 2). One of the conspicuous findings in this study is that IRS-1 is more frequently downregulated in squamous cell carcinoma than in other histologic subtypes ( $p=0.002, \chi^{2}$-test) (Fig. $2 \mathrm{~A}$ and B). We were able to classify 80 tissue samples for histologic grade of differentiation. Thirteen (43.3\%) out of 30-well, 10 (34.5\%) out of 29 moderately, and 9 (42.9\%) out of 21 poorly differentiated NSCLC tissue lost IRS-1 expression and found that IRS-1 expression was not influenced by grade of differentiation. In a subgroup analysis among subtypes of adenocarcinoma, no 
A

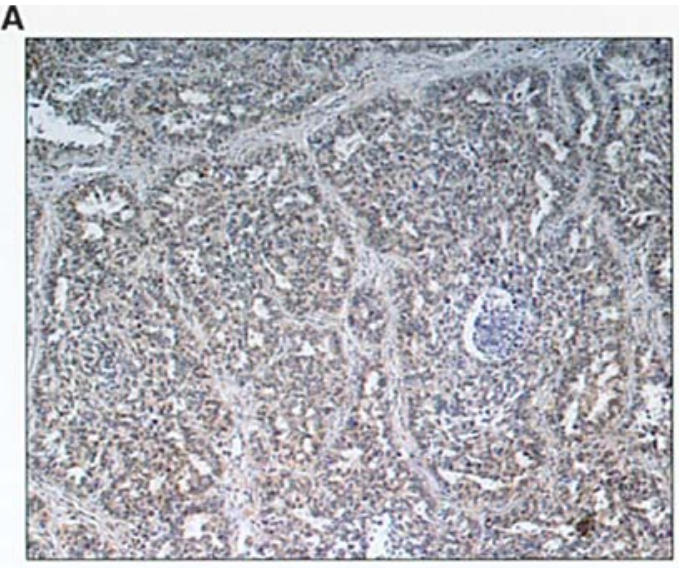

C

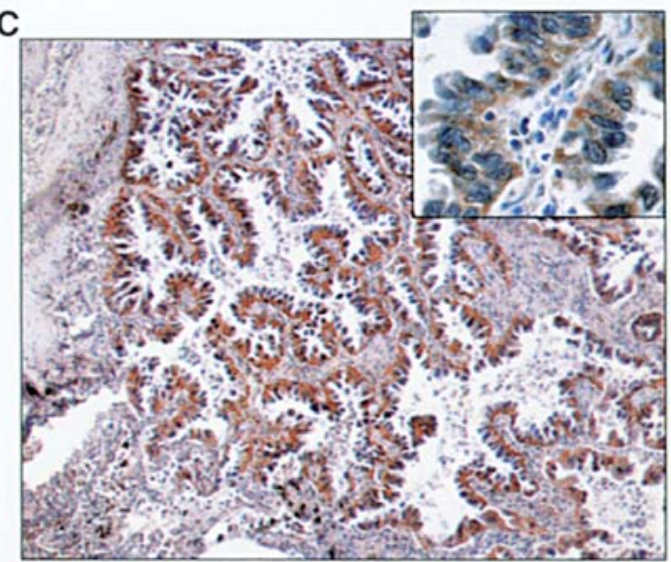

B

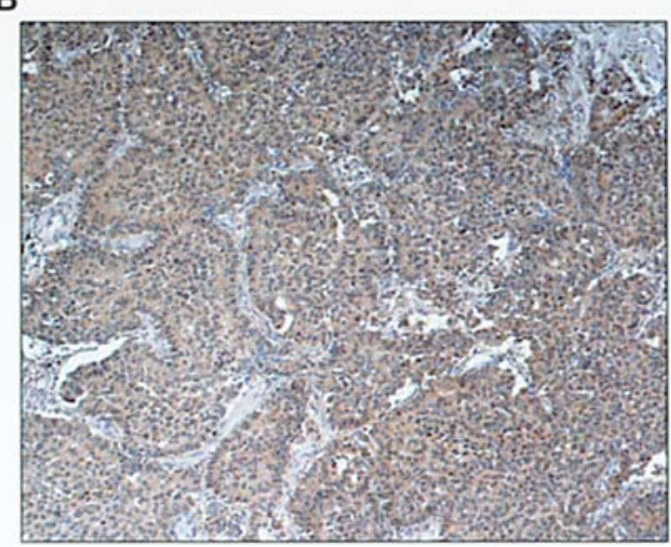

D

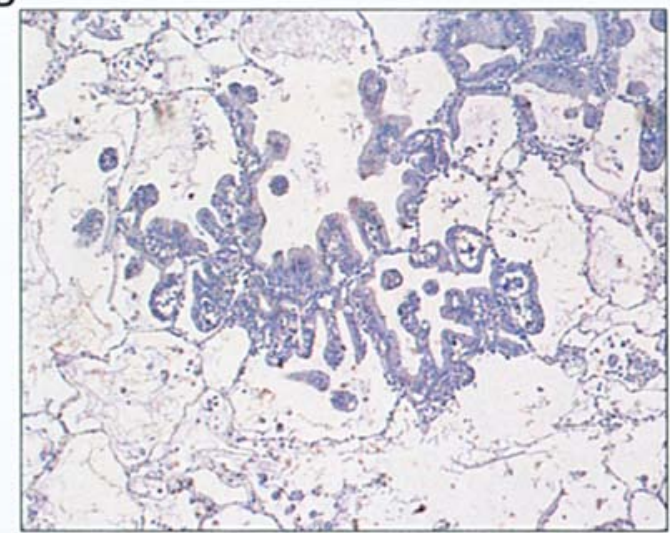

Figure 2. IRS-1 expression in NSCLC tissue. Adenocarcinoma (A) and squamous cell carcinoma (B) of lung showed strong immunoreactivity with IRS-1. A bronchioloalveolar carcinoma has strong IRS-1 expression (C; inset, x400), whereas other do not (D). (Magnification x200).

differences were observed between bronchioloalveolar carcinomas and other subtypes of adenocarcinoma (Fig. 2C and D).

Patient age and gender did not differ between groups whose tumors did and did not exhibit IRS-1 expression. NSCLC tissues from smokers more frequently lost IRS-1 expression than those from non-smokers but this did not reach statistical significance ( $p=0.077, \chi^{2}$ test). Comparing pack-years (P.Y.) between groups of smokers whose tumor expressed IRS-1 and those that did not, smokers whose tumor lost IRS-1 expression had higher P.Y. than those whose tumor did express IRS-1 (39.2 \pm 23.67 vs. $25.6 \pm 26.61$ P.Y., $p=0.034$, ttest). IRS-1 was more frequently down-regulated in the tumor tissues with TNM IB than in those with TNM IA status $\left(50.0\right.$ vs. $22.7 \%, \mathrm{p}=0.024, \chi^{2}$ test). This was reflected by the finding that tumors positive for IRS-1 expression were on average larger than IRS-1-negative tumors (18.1 \pm 14.55 vs. $12.1 \pm 13.76 \mathrm{~cm}^{2}, \mathrm{p}=0.044$, t-test). Table I summarizes the associations between expression status of IRS-1 and clinical and pathological parameters.

Survival analysis. The median follow-up duration of the patients who were alive at the time of analysis was 7.5 years (95\% CI; 7.21-7.86 years). Twenty-nine (30.9\%) out of 94 patients died during the follow-up period and $25(26.6 \%)$ died of cancer-related events. The 5-year survival probability of the entire patient population was $70.1 \%$, which is similar to a previous report from a large-scale study (17). Intratumoral
IRS-1 expression status did not make differences in diseasefree, disease-specific, and overall survival of NSCLC patients (Table I, Fig. 3). Subgroup analyses classified according to pathologic diagnosis, grade of differentiation or TNM stages also showed that IRS-1 expression status did not influence disease-free, disease-specific, and overall survival of NSCLC patients (data not shown).

\section{Discussion}

IGF signaling cascades may be involved in lung cancer development. It is supported by several large scale epidemiologic studies which had shown that high IGF-1 and low IGFBP-3 levels are independent risk factors of lung cancer $(2,18)$. Because IRS- 1 is a major mediator of IGF signaling, we inferred that IRS-1 may play a specific role in NSCLC carcinogenesis or progression. To explore the role of IRS-1 in NSCLC, authors confined study objects to a homogeneous group diagnosed as stage I NSCLC and who underwent curative surgery at the time of diagnosis.

IRS-1 was strongly and consistently expressed in the cytoplasm of intrapulmonary bronchial and bronchiolar epithelial cells comprising normal-appearing lung tissues. Previous studies using normal-appearing breast tissues showed that IRS-1 was strongly to moderately expressed in the cytoplasm of ductal and acinal epithelial cells and that nuclei were sometimes immunoreacted with IRS-1 (16). Our study showed that 


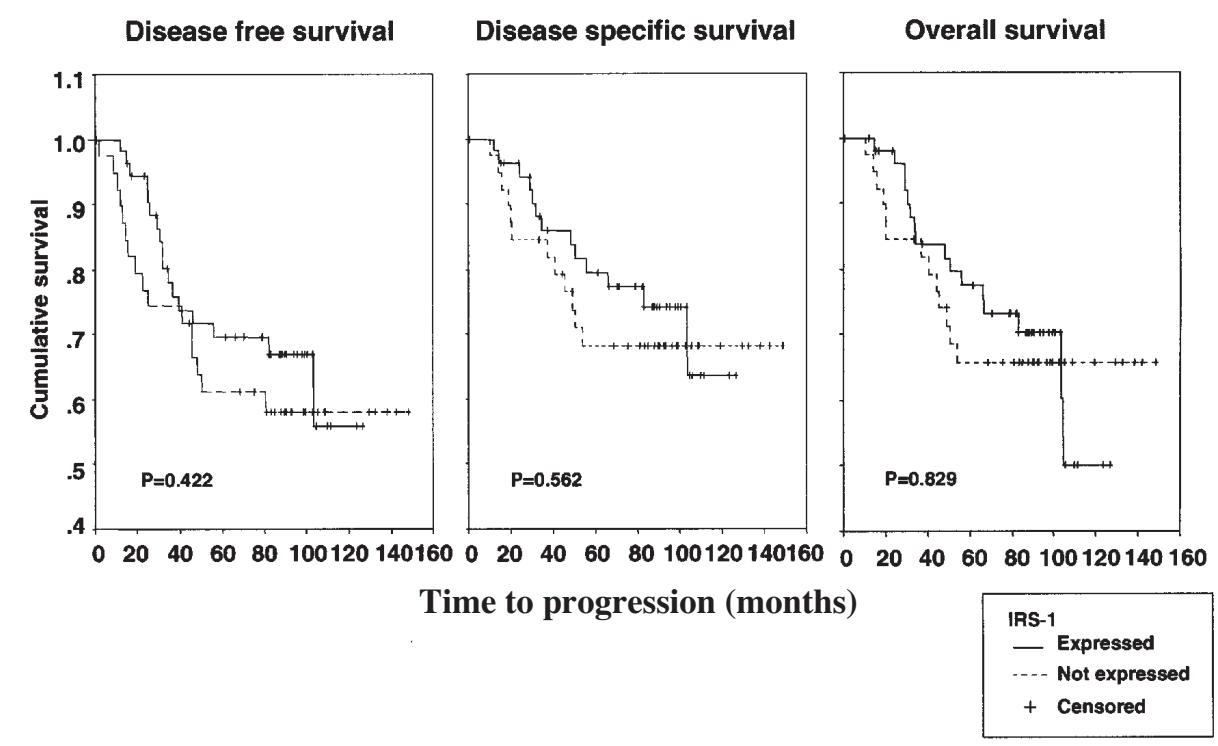

Figure 3. Survival analysis of stage I NSCLC patients according to IRS-1 expression. The intratumoral expression of IRS-1 did not influence disease-free survival (A), disease-specific survival (B) or overall survival (C) of NSCLC patients.

IRS-1 expression was more frequently lost in the NSCLC tissues with larger diameters (T2 tumors) than the smaller ones. The underlying mechanisms for down-regulation of IRS-1 still remain unknown but down-regulation of IRS-1 was also observed in the progression of breast cancer and experimental hepatocarcinogenesis, which indicates that this represents a more general disturbance in signaling during cancer development $(15,16,19)$. Cellular level of IRS-1 is post-translationally modulated by multiple pharmacological reagents, such as retinoic acid derivatives and ubiquitinin proteasome pathway modulators (20). Although physiologic level of circulating IGF-1 protects against degradation of IRS-1 by various stimuli, prolonged exposure to high concentrations of IGF-1, high osmolarity, high glucose, and okadaic acid also accelerate IRS-1 degradation $(10,21)$. Elucidating the mechanisms of IRS-1 down-regulation and finding the materials that regulate expression and/or function of IRS-1 in NSCLC in vivo may be of help for modulation of cancer progression.

There are contradictory reports on the relationship between cancer differentiation and IRS-1 expression. Schnarr et al indicated that well (grade 1) and moderately differentiated (grade 2) ductal carcinoma of breast has moderate to high IRS-1 expression than poorly differentiated ones (16). However, Koda et al reported that IRS-1 is more frequently expressed in the breast cancer tissues with poorly differentiated (grade 3) histology and with lymph node involvement (22). Careful attention must be paid to the fact that Koda et al did not clarify IRS-1 expression in normal adjacent breast tissues and did not compare intratumoral IRS-1 expression with that in normal adjacent tissues. Our study revealed that $43 \%$ of well, $34 \%$ of moderately and $42 \%$ of poorly differentiated NSCLC tissues showed loss of IRS-1 expression and suggest that histologic grade of differentiation did not influence IRS-1 expression in NSCLC tissue.

IRS-1 was more frequently lost in squamous cell carcinoma of the lung than adenocarcinoma. The underlying mechanisms for more frequent loss in squamous cell carcinoma is unknown but it may be partially supported by earlier studies investigating the consequences of IGF and IGF receptor dysfunction in lung cancer. Pavelic et al suggested a correlation between increased expression for IGF-1 and IGF$1 \mathrm{R}$ that has protective property against IRS-1 degradation, as well as decreased apoptosis in adenocarcinomas (23).

The significance of IRS-1 expression for prognosis in cancers has been a matter of debates because of conflicting results (16). High IRS-1 level, which is determined by ELISA or immonoblotting, were associated with early breast cancer recurrence $(24,25)$. We confined the study to the homogeneous cases, and showed that intratumoral IRS-1 expression did not influence the prognosis of patients when analyzed in terms of disease-free, disease-specific and overall survival. Taken together, intratumoral down-regulation of IRS-1 is suggested to be a molecular event related to the early development of NSCLC rather than a prognostic factor. Down-regulation of IRS-1 might be of help for classification of NSCLC because it shows a more frequent loss in squamous cell carcinoma than other cell types. More comprehensive studies of the mechanisms that induce loss of IRS-1 expression in NSCLC are necessary for the elucidation of the role of IRS-1 in lung carcinogenesis. Insights gained from this basic study may lead to novel clinical research targeted at prevention and therapy of NSCLC.

\section{Acknowledgements}

This study was supported by a Korea Research Foundation Grant funded by the government of the Republic of Korea (MOEHRD, Basic Research Promotion Fund, KRF-2005-003E00101) and by the Korea Science and Engineering Fund through the Cancer Metastasis Research Center at Yonsei University, Seoul, Korea.

\section{References}

1. Le Roith D: Seminars in medicine of the Beth Israel Deaconess Medical Center. Insulin-like growth factors. N Engl J Med 336: 633-640, 1997. 
2. Yu H, Spitz MR, Mistry J, Gu J, Hong WK and Wu X: Plasma levels of insulin-like growth factor-I and lung cancer risk: a casecontrol analysis. J Natl Cancer Inst 91: 151-156, 1999.

3. Chan JM, Stampfer MJ, Giovannucci E, Gann PH, Ma J, Wilkinson P, Hennekens CH and Pollak M: Plasma insulin-like growth factor-I and prostate cancer risk: a prospective study. Science 279: 563-566, 1998.

4. Hankinson SE, Willett WC, Colditz GA, Hunter DJ, Michaud DS, Deroo B, Rosner B, Speizer FE and Pollak M: Circulating concentrations of insulin-like growth factor-I and risk of breast cancer. Lancet 351: 1393-1396, 1998

5. Ma J, Pollak MN, Giovannucci E, Chan JM, Tao Y, Hennekens CH and Stampfer MJ: Prospective study of colorectal cancer risk in men and plasma levels of insulin-like growth factor-I and IGF binding protein-3. J Natl Cancer Inst 91: 620-625, 1999.

6. Tsuruzoe K, Emkey R, Driaucinunas KM, Ueki K and Kahn CR: Insulin receptor substrate-3 (IRS-3) and IRS-4 impair IRS-1and IRS-2-mediated signaling. Mol Cell Biol 21: 26-38, 2001.

7. Gual P, Le Marchand-Brustel Y and Tanti JF: Positive and negative regulation of insulin signaling through IRS-1 phosphorylation. Biochimie 87: 99-109, 2005.

8. Delafontaine P, Song YH and Li Y: Expression, regulation and function of IGF-1, IGF-1R and IGF-1 binding proteins in blood vessels. Arterioscler Thromb Vasc Biol 24: 435-444, 2004.

9. Esposito DL, Li Y, Cama A and Quon MJ: Tyr(612) and $\operatorname{Tyr}(632)$ in human insulin receptor substrate-1 are important for full activation of insulin-stimulated phosphatidylinositol 3-kinase activity and translocation of GLUT4 in adipose cells. Endocrinology 142: 2833-2840, 2001

10. Kim B, Oh S, van Golen CM and Feldman EL: Differential regulation of insulin receptor substrate-1 degradation during mannitol and okadaic acid induced apoptosis in human neuroblastoma cells. Cell Signal 17: 769-775, 2005

11. Morelli C, Garofalo C, Bartucci M and Surmacz E: Estrogen receptor-alpha regulates the degradation of insulin receptor substrates 1 and 2 in breast cancer cells. Oncogene 22: 4007-4016, 2003.

12. Yenush L and White MF: The IRS-signalling system during insulin and cytokine action. Bioessays 19: 491-500, 1997.

13. Burks DJ and White MF: IRS proteins and beta-cell function. Diabetes 50 (Suppl. 1): S140-S145, 2001.

14. Reiss K, Wang JY, Romano G, Furnari FB, Cavenee WK, Morrione A, Tu X and Baserga R: IGF-I receptor signaling in a prostatic cancer cell line with a PTEN mutation. Oncogene 19: 2687-2694, 2000.

15. Nehrbass D, Klimek F, Bannasch P and Mayer D: Insulin receptor substrate-1 is overexpressed in glycogenotic but not in amphophilic preneoplastic hepatic foci induced in rats by N-nitrosomorpholine and dehydroepiandrosterone. Cancer Lett 140: 75-79, 1999.
16. Schnarr B, Strunz K, Ohsam J, Benner A, Wacker J and Mayer D Down-regulation of insulin-like growth factor-I receptor and insulin receptor substrate-1 expression in advanced human breast cancer. Int J Cancer 89: 506-513, 2000.

17. Martini N, Bains MS, Burt ME, Zakowski MF, McCormack P, Rusch VW and Ginsberg RJ: Incidence of local recurrence and second primary tumors in resected stage I lung cancer. J Thorac Cardiovasc Surg 109: 120-129, 1995.

18. Lee CT, We S, Gabrilovich D, Chen H, Nadaf-Rahrov S, Ciernik IF and Carbone DP: Antitumor effects of an adenovirus expressing antisense insulin-like growth factor-1 receptor on human lung cancer cell lines. Cancer Res 56: 3038-3041, 1996.

19. Nehrbass D, Klimek F and Bannasch P: Overexpression of insulin receptor substrate-1 emerges early in hepatocarcinogenesis and elicits preneoplastic hepatic glycogenosis. Am J Pathol 152: 341-345, 1998.

20. Del Rincon SV, Guo Q, Morelli C, Shiu HY, Surmacz E and Miller WH: Retinoic acid mediates degradation of IRS-1 by the ubiquitin-proteasome pathway, via a PKC-dependant mechanism. Oncorgene 23: 9269-9279, 2004.

21. Pederson TM, Kramer DL and Rondinone CM: Serine/threonine phosphorylation of IRS-1 triggers its degradation: possible regulation by tyrosine phosphorylation. Diabetes 50: 24-31, 2001.

22. Koda M, Sulkowska M, Kanczuga-Koda L and Sulkowski S: Expression of insulin receptor substrate 1 in primary breast cancer and lymph node metastases. J Clin Pathol 58: 645-649, 2005.

23. Pavelic J, Krizanac S, Kapitanovic S, Pavelic L, Samarzija M, Pavicic F, Spaventi S, Jakopovic M, Herceg-Ivanovi Z and Pavelic K: The consequences of insulin-like growth factors/ receptors dysfunction in lung cancer. Am J Respir Cell Mol Biol 32: 65-71, 2005

24. Lee AV, Jackson JG, Gooch JL, Hilsenbeck SG, CoronadoHeinsohn E, Osborne CK and Yee D: Enhancement of insulinlike growth factor signaling in human breast cancer: estrogen regulation of insulin receptor substrate-1 expression in vitro and in vivo. Mol Endocrinol 13: 787-796, 1999.

25. Rocha RL, Hilsenbeck SG, Jackson JG, van den Berg CL, Weng C, Lee AV and Yee D: Insulin-like growth factor binding protein-3 and insulin receptor substrate-1 in breast cancer: correlation with clinical parameters and disease-free survival. Clin Cancer Res 3: 103-109, 1997. 